\title{
Editorial \\ Vaginal hysterectomy: step ladder technique without postoperative vault prolapse
}

Volume 6 Issue 3 - 2017

\section{Editorial}

Hysterectomy is one of the most practiced gynecologic operations. In spite of the enthusiasm for laparoscopic and robotic techniques, I feel that vaginal route is the best over the abdominal, laparoscopic and even robotic techniques. Training of the technique is simple, add to that, it is the winner regarding the cost, complications and hospital stay.

Vaginal vault prolapse is one of the most frustrating complications and the saying "prevention is better than cur" is well applicable in that context. Sir victor Bonney said; the possibility of curing a case of prolapse after hysterectomy without narrowing the vagina, preventing sexual relations is about to be non-existent. ${ }^{1}$ This explains the surge of papers for correction of such a problem. The index work puts some stress on preventing such a complication by some modifications of the technique of vaginal hysterectomy. Vaginal vault prolapse is either due to loss of normal pelvic support or to omitting the steps that benefit of these supportive structures during the operation. Post hysterectomy vaginal vault prolapse could be prevented during hysterectomy. ${ }^{2}$

\section{Suggested modifications of the technique}

After pushing up the bladder and opening the Pouch of Douglas (POD), the 1st clamp is applied to uterosacral ligament as close to the uterus as possible; confirming that the inside blade is inside the peritoneal cavity to include the small vessels between the peritomeum and the base of the pelvis. Being close to the uterus; avoid damage to surrounding structures especially the angle of the bladder that might be caught down and gives a good pedicle. We need long pedicle here as one of the main difficulties to encounter is the lack of mobility of the uterus in the early steps of the operation that makes the field tight. With good pedicle gives safeguard if the clamp have slipped.

First ligatures is left with long threads, one with needle will be used to have a bite in the lateral vaginal angle so: Support the vaginal vault by ligating it to the main supporting structures of the pelvis. Shares in the hemostasis of that vascular area.

Almost always the $2^{\text {nd }}$ bite (Clamp) will not reach the level of uterine vessels and we don't intend to do so. I think that if one bite is taken to the whole uterosacral ligament, it would be a big pedicle that necessitates more than one suture ligature to control too big pedicle. Usually 2 bites are needed to finish the whole uterosacral ligament.

The long thread of the $1^{\text {st }}$ bite is tied with one of the threads of the next ligature so the whole uterosacral was at the end taken to the vaginal angle. At the same time ligating one thread from each bite to the next will occlude many small vessels in between. One of the threads will be left long and attached to a marker clamp, the other will be tied to one of the threads of uterine pedicle. The marker thread will help in:

1. If there is slippage of the pedicle, gentle traction on that marker will help to bring the whole suture area down, so it is used for traction if needed.

\author{
Galal Lotfi \\ Obstetrics \& gynecology Department, Suez Canal University, \\ Egypt
}

Correspondence: Galal Lotfi, Obstetrics \& gynecology Department, Faculty of Medicine, Suez Canal University, Ismaila, Egypt, Email glotif@gmail.com

Received: January 26, 2017| Published: March 03, 2017

\section{It will be ligated to the ovarian pedicle.}

So, at the end of operation we find that the whole three pedicles are ligated together on one side with marked stitch. During peritonization, one thread from round ligament was tied to its counterpart on the other side and peritoneum was approximated and at the end of peritonization, stitches was tied to one of the marker stitches. The two marker stitches were tied together and the long marker thread was used to close the peritoneal vault. So, the whole vault was supported by the uterosacral. In some patients peritonization was almost impossible because of tight vagina or high POD and it was ignored.

The cardinal ligament (Mackenrodts \& uterosacral) arise from the lateral aspect of the vagina rather than from the Uterus. These ligaments afford the main support of the uterus and upper vagina. ${ }^{3}$ For that reason the proposed modifications entails suturing of the cardinal ligament and lateral vaginal wall together to create adhesive forces that help holding the vagina.. Because posthysterectomy vaginal vault prolapse occurs after transvaginal and transabdominal hysterectomy, it goes without saying that hysterectomy alone does not cure or prevent genital prolapse. Vaginal route gives the gynecologist the opportunity to visualize and use the pelvic support and attach them to the vaginal vault. The suggested technique calls attention to attaching the cardinal ligament to the vagina that aids in preventing post hysterectomy vaginal vault prolapse or enterocele as recommended by Cruikshank. ${ }^{4}$

So, as the whole pedicles on one side are attached to the vaginal angle, that helps in:

a. The marker stitch can help in pulling down any part of any pedicle when bleeding point has to be secured.

b. Ligaturing the pedicles together will occlude the small vessels in between making good hemostasis.

c. Approximating uterosacral of both sides give good support to the vagina preventing posthysterectomy vaginal vault prolapse.

So, in support we are using cardinal ligament and at the same time we use the round ligament. It is to be mentioned that hysterectomy 
alone does not take care of the vault prolapse. Precautions during the operation by using the main uterovaginal support help to prevent the occurrence of such complication when some precipitating factors are on the way e.g. postmenopausal weakness of the ligament.

It should be noticed that the vagina lies parallel to the levatorani a horizontal position. With increased intrabdominal pressure, the levator ani and cardinal ligament hold the cervix and upper vagina in their proper position. Distortion of vaginal axis due to weak support places the vaginal apex in an unsupported position over the uterovaginal hiatus i.e. the vagina cannot rest upon the levator plate resulting in prolapse. This illustrates the importance of normal; vaginal axis in the integrity of the vaginal support. In the technique described the round ligament was attached to the cardinal ligament. Some authors used the round ligament as part of vaginal fixation ${ }^{5,6}$ to prevent post hysterectomy vaginal vault prolapse. I feel that the round ligament in that issue is trivial, however the used technique of attaching the round ligament to the cardinal ligament help to make peritonozation easier. This conclude that vaginal hysterectomy should be taken as an opportunity to prevent posthysterectomy vaginal vault prolapse by meticulous care of supporting ligaments. ${ }^{7,8}$

\section{Conclusion}

The proposed modified technique helps in prevention of posthysterectomy vaginal vault prolapse as it assists in keeping the vaginal axis in its normal situation parallel to the levator ani. The step above step suturing is important to keep any pedicles in quick reach once it becomes necessary to do so. The technique helps to prevent the tragic abdomino-vaginal approach; if bleeding pedicle is retracted out of reach and the frustrating posthysterectomy vaginal vault prolapse. Vaginal hysterectomy is a safe and skilled technique that should be considered during assessment of every patient planned to have hysterectomy.

\section{Acknowledgments}

None.

\section{Conflicts of interest}

None.

\section{References}

1. Berkeley C, Bonney V. Gynecologic surgery. P Hoeber Inc, New York, USA. 1948;pp.203.

2. Kaser O, Ikle FA, Hirsch HA. Atlas of gynecologic surgery. (2nd edn), Thieme-Straton, New York, USA. 1985;p.61-64.

3. Howkins J, Bourne C. Anatomy of the pelvis. In: Howkins J \& Bourne G (Eds.), Shaw's textbook of gynecology. Churchill Livingstone, Edinburgh, UK. 1976;pp.510.

4. Cruikshank SH. Preventing post hysterectomy vaginal vault prolapse and enterocele during vaginal hysterectomy. Am J Obstet Gynecol. 1987;155(6):1433-1440.

5. Symmonds RE, Williams TJ, Lee RA, et al. Post hysterectomy enterocele and vaginal vault prolapse. Am J Obstet Gynecol. 1981;140(8):852-859.

6. Randall CL, Nichols DH. Surgical treatment of vaginal inversion. Obstet . 1971;38(3):327-332.

7. Howkins $\mathrm{J} \&$ Stallworthy J.Vaginal hysterectomy. In: Howkins $\mathrm{J} \&$ Stallworthy J (Eds.), Bonney Gynecologic surgery. Williams Clowes \& sons limited. London. 1974;pp.227-254.

8. Mattingl RF, Thompson JD. Telinde's operative gynecology. (6th edn), JB Lappincott, Philadelphia, USA. 1983;pp.555-559. 